\title{
Insulin growth factors may explain relationship between spasticity and skeletal muscle size in men with spinal cord injury
}

\author{
Ashraf S. Gorgey, MPT, PhD, FACSM; David R. Gater, MD, PhD \\ Spinal Cord Injury and Disorders Center, Hunter Holmes McGuire Department of Veterans Affairs Medical Center, \\ Richmond, VA; Department of Physical Medicine and Rehabilitation, Virginia Commonwealth University, \\ Richmond, VA
}

\begin{abstract}
The major objectives of this cross-sectional study were to (1) measure insulin-like growth factor-1 (IGF-1) in individuals with complete spinal cord injury (SCI) and spasticity and (2) determine the relationships between IGF-1 and cross-sectional areas (CSAs) of thigh skeletal muscle groups. Eight individuals with motor complete SCI underwent magnetic resonance imaging to measure the CSA of the whole thigh, knee extensor, and knee flexor skeletal muscle groups and dual-emission X-ray absorptiometry to measure fat-free mass. After participants fasted for $12 \mathrm{~h}$, we measured their IGF-1 levels and determined spasticity using the Modified Ashworth Scale (MAS). Spearman rho correlations were used to test for the relationships among the tested variables, and independent $t$-tests were used to determine the difference in plasma IGF-1. Plasma IGF-1 was $44 \%$ greater in those with MAS scores of 2 or higher $(p<0.05)$. Plasma IGF-1 was positively related to knee extensor skeletal muscle CSA $(r=0.83$, $p<0.01)$. IGF-1 was strongly related to knee extensor and flexor spasticity $(r=0.88, p<0.004)$. The findings suggest that IGF-1 is greater in SCI individuals with increased spasticity, and this may explain the strong positive relationships that were noted between spasticity and skeletal muscle CSA.
\end{abstract}

Key words: body composition, growth hormone, IGF-1, insulin-like growth factor, level of injury, Modified Ashworth Scale, MRI, rehabilitation, SCI, skeletal muscle, spasticity.

\section{INTRODUCTION}

Individuals with motor complete spinal cord injury (SCI) typically present with loss of motor control, dimin- ished superficial and deep sensation, and exaggerated spasticity below the level of injury [1-2]. Extreme immobilization and disuse may ensue, with dramatic skeletal muscle atrophy, altered body composition, and deranged metabolic profile [3-5]. SCI is characterized by diminished growth hormone (GH), insulin-like growth factor-1 (IGF-1), and testosterone; the decline in anabolic hormones may further exacerbate muscle atrophy and fat mass (FM) gain below the level of injury [6-7]. Despite continuous research efforts to understand the factors that either contribute to or attenuate the aforementioned profile, there is still an apparent gap that needs to be further addressed to physiologically explain the adaptations after SCI. This knowledge could be translated into effective interventions that could be of significant importance to this population.

\footnotetext{
Abbreviations: $\mathrm{BMI}=$ body mass index, $\mathrm{CSA}=$ cross-sectional area, $\mathrm{FFM}=$ fat-free mass, FM = fat mass, $\mathrm{GH}=$ growth hormone, IGF-1 = insulin-like growth factor-1, MAS = Modified Ashworth Scale, RNA = ribonucleic acid, SCI $=$ spinal cord injury.

*Address all correspondence to Ashraf S. Gorgey, MPT, PhD, FACSM; Hunter Holmes McGuire Department of Veterans Affairs Medical Center, Spinal Cord Injury and Disorders Service, 1201 Broad Rock Blvd, Richmond, VA 23249; 804-675-5000, ext 3603/3386; fax: 804-675-5223.

Email: ashraf.gorgey@va.gov

http://dx.doi.org/10.1682/JRRD.2011.04.0076
} 
Spasticity is a characteristic of upper motor neuron lesions and primarily evolves as a result of the loss of the inhibitory supraspinal control on the stretch reflex [2]. Despite spasticity's negative effects, several reports have found that it has a positive influence on skeletal muscle size; intramuscular fat; body composition; and metabolic profile, including carbohydrate and lipid profiles [8-13]. We have previously shown that spastic muscle has a 22 percent larger cross-sectional area (CSA) than nonspastic muscle in individuals with incomplete SCI [8]. This observation was also confirmed by another group that studied the effects of spasticity on bone mineral density [12]. Furthermore, in a cross-sectional study, we identified inverse relationships between knee extensor spasticity and both truncal and whole body FM [9]. The trial documented positive relationships between spasticity and lean mass, which have played a protective role against reduction in resting energy expenditure, hyperlipidemia, and impaired glucose tolerance [9]. Others have documented that spastic muscles have three times greater glucose sensitivity than nonspastic controls [11].

In an effort to understand the mechanism(s) behind the protective effects of spasticity, researchers previously noted a similarity between the involuntary periodical tension developed during spasticity and resistance training [8,13-14]. Resistance training is responsible for evoking skeletal muscle hypertrophy and improving body composition through its effects on IGF-1 production [14-15]. We recently documented a 25 percent increase in plasma IGF-1 following 12 weeks of electrical stimulation resistance training in persons with motor complete SCI; the increase was associated with a decrease in ectopic adipose tissue and a 10 percent increase in leg lean mass [16]. A recent report showed that dorsal rhizotomy accompanied by denervation and loss of spasticity evoke greater muscle loss than spinal isolation in rats because of the upregulation in muscle-specific ubiquitin-protein ligases [17]. The authors suggested that the catabolic atrophic factors downstream the IGF-1 pathway [17]. We are unaware of any reports that have identified the effects of spasticity on IGF-1 in men with SCI. The primary purpose of the current study was to establish the relationships between spasticity and IGF-1 as well as muscle size and IGF-1 in persons with motor complete SCI. We hypothesized that spasticity could defend against muscle atrophy and deleterious changes in body composition through its effects on IGF-1.

\section{METHODS}

\section{Study Design and Participants}

"Eight, healthy, males with chronic traumatic motor complete SCI ([cervical] 5 to [thoracic] 11), mean \pm SD [standard deviation] age $35 \pm 9$ years and BMI [body mas index] $22 \pm 4 \mathrm{~kg} / \mathrm{m}^{2}$ ) participated in the study. Participants were required to be at least one year post-injury between $18-50$ years old, BMI $\leq 30 \mathrm{~kg} / \mathrm{m}^{2}$, wheelchair reliant, with traumatic motor complete C5-T11 level of injury, American Spinal Injury Association Impairment Scale (AIS) A and B; i.e., complete motor paresis below the level of injury" [16]. For 1 yr, participants should have maintained the same dose of anti-spasticity medication to ensure stability of spasticity at the time of measurements. Similar to previous published work [16,18], "participants with pre-existing medical conditions were excluded (cardiovascular diseases, type II diabetes, pressures sores greater than stage 2). Recruitment was done by word of mouth and by approved flyers from the community venues of University Hospital” [16].

"Participants were instructed to abstain from exercise, as well as alcohol and caffeine consumption $24 \mathrm{~h}$ preceding the exam. Upon arrival, they were asked to void their bladder and undergo a general physical examination that included vital sign collection and a resting 12lead electrocardiogram to rule out any pre-existing cardiac problems. Body weight was measured while wearing light clothes in a supine position using a hospital bed scale calibrated to $0.1 \mathrm{~kg}$. Height was measured from the same position to the nearest $0.1 \mathrm{~cm}$. BMI was calculated as weight in [kilograms] divided by height in [meters squared]" [17].

\section{Magnetic Resonance Imaging and Body Composition}

The technique was previously described in detail elsewhere [16]. Briefly, "magnetic resonance images were obtained with a General Electric [Fairfield, Connecticut] Signa 1.5-T magnet (repetition time $550 \mathrm{~ms}$; echo time $14 \mathrm{~ms}$; field of view $20 \mathrm{~cm}$; matrix size $256 \times$ 256) $[4,8]$. Whole thigh, knee extensors, and flexors skeletal muscle CSAs were measured. Transaxial images, $10 \mathrm{~mm}$ thick and $5 \mathrm{~mm}$ apart, were taken from the hip joint to the knee joint using the whole body coil. Before starting the scan, both legs were strapped together to avoid involuntary spasm movements and participants were provided with earplugs to minimize the magnet noise. 
"Images were downloaded and analyzed using Win Vessels program (Ronald Meyer, [Michigan State University]) as has been previously described. For skeletal muscle and IMF [intramuscular fat] CSAs, the outer perimeter of the thigh muscle groups was traced and pixel signal intensity within this region was automatically determined. Dual energy X-ray absorptiometry (Hologic QDR-2000 scanner; [Bedford, Massachusetts]) was used to quantify whole body fat-free mass (FFM) ([kilograms]) [9]" [16]. Whole body FFM was used to adjust for differences in body size among participants.

\section{Modified Ashworth Scale}

The Modified Ashworth Scale (MAS) (Table 1) was used to evaluate spasticity of hip, knee, and ankle skeletal muscle flexors and extensors and hip adductor muscles for both lower limbs in the morning (8:00 a.m.) [4,8-10]. The room temperature was held constant at $21^{\circ} \mathrm{C}$ to $24^{\circ} \mathrm{C}$. After participants lay flat in a hospital bed for $30 \mathrm{~min}$, their spasticity was evaluated in a supine position by the same investigator. Participants were instructed not to use a pillow and to keep the head in a neutral position during the test. Spasticity was evaluated for the primary muscle groups of both lower limbs, as was previously performed [9]. Each major muscle group was examined three times before being assigned a final score. Similar to previous works [9-10], a spasticity composite score of the sum of flexor (hip, knee, and ankle dorsiflexors) and extensor (hip, knee, and ankle plantar flexors) primary muscle groups was used to determine the relationship(s) of whole limb tonicity to other dependent variables. Knee flexor spasticity was measured to account for the scien-

Table 1.

Modified Ashworth Scale score definitions.

\begin{tabular}{|c|c|}
\hline Score & Definition \\
\hline 0 & No increase in skeletal muscle tone. \\
\hline 1 & $\begin{array}{l}\text { Slight increase in skeletal muscle tone manifested by } \\
\text { catch and release at end of range of motion. }\end{array}$ \\
\hline 2 & $\begin{array}{l}\text { Slight increase in skeletal muscle tone, manifested by } \\
\text { catch, followed by minimal resistance throughout range } \\
\text { of motion. }\end{array}$ \\
\hline 3 & Considerable increase in skeletal muscle tone. \\
\hline 4 & $\begin{array}{l}\text { More marked increase in skeletal muscle tone through- } \\
\text { out range of motion. }\end{array}$ \\
\hline 5 & Rigid in flexion or extension. \\
\hline
\end{tabular}

tific bias that adaptation in body composition is only related to knee extensor spasticity. We started by testing the muscle groups in the right leg and then examining the corresponding muscle groups in the left leg.

During the same visit and after participants fasted overnight for $12 \mathrm{~h}$, we inserted a Teflon catheter into an antecubital vein of their right arm for blood sampling. Three blood samples were collected at $15 \mathrm{~min}$ intervals for assessment of total plasma IGF-1. To examine the effects of spasticity on IGF-1 profile, we used a knee extensor MAS cutoff score of $\geq 2$ to separate participants into two groups: MAS score $\geq 2(n=5)$ and MAS score $<2(n=3)$. The cutoff was based on evidence that a MAS score $\geq 2$ is essential to defend against muscle atrophy [8].

\section{Statistical Analyses}

Given the ordinal nature of MAS, Spearman $\rho$ correlation was used to determine the relationships among spasticity, IGF-1, and skeletal muscle size. In studying the relationship between spasticity and skeletal muscle CSA, we treated each leg as an independent variable instead of averaging the scores between the right and left legs ( $n=16$ legs) because each muscle group was independently evaluated. Independent $t$-tests were used to test the effects of spasticity on plasma IGF-1 based on the predetermined MAS cutoff. Statistical analyses were performed using SPSS version 17.0 (SPSS Inc; Chicago, Illinois), and all values are presented as mean \pm standard deviation.

\section{RESULTS}

The MAS scores of the major muscle groups are presented in Table 2. Knee extensor spasticity was positively related to knee extensor skeletal muscle CSA ( $r=$ $0.63, p=0.008$; Figure 1(a)) and whole thigh skeletal muscle CSA ( $r=0.49, p=0.049$; Figure 1(b)). After we adjusted thigh CSA to whole body FFM, a trend for positive correlation still existed with knee extensor spasticity ( $r=0.47, p=0.06$ ), suggesting that whole body lean mass does not influence the relationship between spasticity and muscle size.

The average plasma IGF-1 was 43 percent higher in those with MAS scores $\geq 2$ than in those with MAS scores $<2(239 \pm 67$ vs $135 \pm 5 \mathrm{ng} / \mathrm{mL}, p=0.04)$. Note that percentage variability across the three $15 \mathrm{~min}$ interval samples was 15 percent, with a range of 0.06 to 37 percent. 
Table 2.

Modified Ashworth Scale (MAS) scores for different skeletal muscle groups in individuals with motor complete spinal cord injury.

\begin{tabular}{lrr}
\hline \multicolumn{1}{c}{ Muscle Group } & $\begin{array}{c}\text { Right } \\
\text { Lower Limb }\end{array}$ & $\begin{array}{c}\text { Left } \\
\text { Lower Limb }\end{array}$ \\
\hline Hip Flexor $^{*}$ & $2.1 \pm 1.1$ & $2.0 \pm 0.9$ \\
Hip Extensor $^{\dagger}$ & $2.5 \pm 1.2$ & $2.5 \pm 1.4$ \\
Hip Adductor $^{*}$ & $2.4 \pm 1.2$ & $2.0 \pm 1.1$ \\
Knee Flexor $^{\dagger}$ & $2.1 \pm 1.1$ & $2.1 \pm 1.1$ \\
Knee Extensor $^{\dagger}$ & $2.4 \pm 1.2$ & $2.4 \pm 1.8$ \\
Ankle Dorsiflexor $^{*}$ & $2.3 \pm 1.0$ & $2.0 \pm 0.9$ \\
Ankle Plantar Flexor $^{\dagger}$ & $2.6 \pm 1.3$ & $2.6 \pm 1.2$ \\
Flexor Spasticity $^{\dagger}$ & $6.5 \pm 3.0$ & $6.1 \pm 2.8$ \\
Extensor Spasticity & $7.5 \pm 3.4$ & $7.5 \pm 3.7$ \\
\hline
\end{tabular}

Note: Values are mean \pm standard deviation.

*Flexor spasticity is sum of three MAS scores at hip, knee, and ankle flexor muscle groups.

${ }^{\dagger}$ Extensor spasticity is sum of three MAS scores at hip, knee, and ankle extensor muscle groups.

Strong positive relationships were noted among plasma IGF-1, knee extensor spasticity $(r=0.83, p=$ $0.01)$, and knee extensor CSA ( $r=0.83, p=0.01$ ) as presented in Figure 2. Note that the sum of flexor spasticity $(r=0.77, p=0.02)$ and the sum of extensor spasticity $(r=$ $0.88, p=0.004$ ) of the major muscle groups were positively associated with plasma IGF-1 concentration profile.

\section{DISCUSSION}

The protective effects of spasticity against skeletal muscle atrophy, altered body composition, and deranged metabolic profile after SCI have been previously established [8-10]. The current study was undertaken to unravel the physiological mechanism by which spasticity exerts its protective effects. The major finding was that participants with MAS scores $\geq 2$ had 44 percent higher circulating plasma IGF-1 than those with lower MAS scores. Moreover, elevated plasma IGF-1 was positively related to both spasticity and skeletal muscle CSA; the relationship was still evident after adjusting for whole body FFM.

SCI leads to several deleterious pathological adaptations similar to disuse atrophy, including increased conversion to fast-twitch fatigable fibers, decreased lean mass and increased FM, and altered metabolic profile [1-5]. Studying factors that could attenuate this phenotype may lead to reduced comorbidities and mortalities after SCI. Spasticity is a velocity-dependent phenomenon charac-
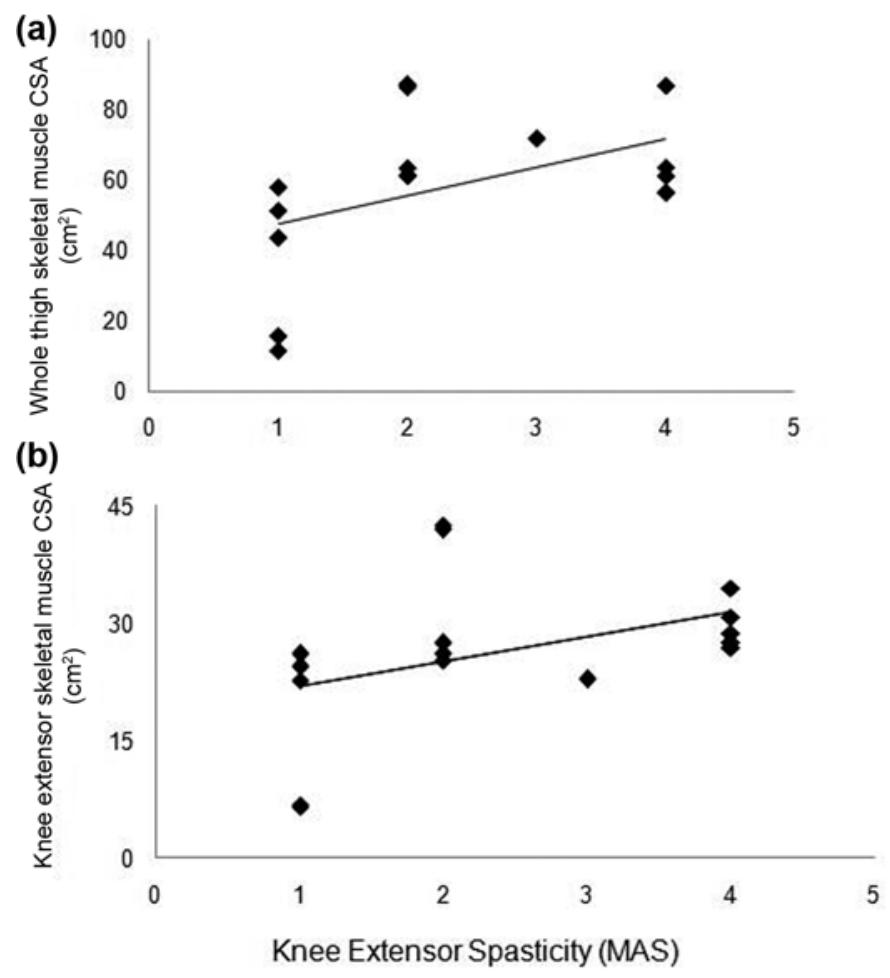

Figure 1.

Relationship between knee extensor spasticity (as measured by Modified Ashworth Scale [MAS]) and (a) whole thigh skeletal muscle cross-sectional area (CSA) and (b) knee extensor skeletal muscle CSA. Positive relationships were identified as highlighted in "Results" section of main text.

terized by increased muscle tone in response to passive movement. Spasticity has been previously shown to preserve slow-to-fast fiber conversion in the tail muscle of SCI rats [19]. As early as 6 weeks post-SCI, spasticity appears to exert a protective effect on skeletal muscle size and composition in those with a high level of injury compared with nonspastic individuals with a low level of injury [8]. Others have documented that glucose uptake is three times higher in spastic muscles than in controls [11].

A fundamental question that remains unanswered is how periodic involuntary muscle tone could turn off the deleterious effects of SCI. Following SCI, associated reductions in anabolic hormones such as testosterone and GH occur [6-7]. GH release is blunted and chronically depressed in SCI, as evidenced by reduced levels of IGF1 , a convenient indicator of chronic GH secretion [6-7]. In a provocative study using argenine to stimulate $\mathrm{GH}$ release in SCI twins with paraplegia, no difference in 

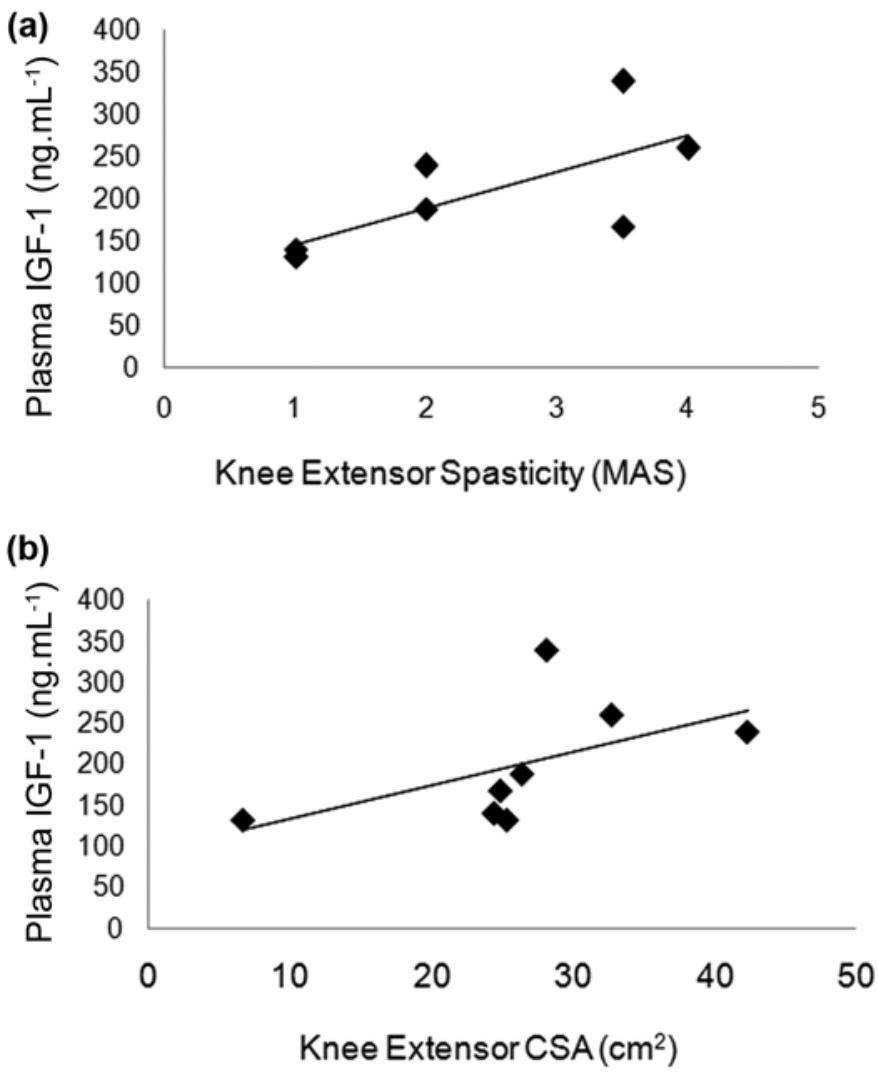

Figure 2.

Relationship between plasma insulin-like growth factor-1 (IGF-1) concentration and (a) knee extensor spasticity (as measured by Modified Ashworth Scale [MAS]) and (b) knee extensor skeletal muscle cross-sectional area (CSA). Positive relationships were identified as highlighted in "Results" section of main text.

IGF-1 profile between groups was noted despite a significant difference in $\mathrm{GH}$ profile [7]. A possible explanation is that spasticity maintained the IGF-1 profile despite lower GH. This observation is confirmed by another finding that clearly documented that persons with poliomyelitis have diminished IGF-1 profiles [20]. Persons with poliomyelitis commonly experience flaccid paralysis due to injury of their alpha motor neurons.

The source of plasma IGF-1 is still debatable; IGF-1 is released from the liver and extrahepatic tissues in response to GH. Yakar et al. noticed that extrahepatic IGF-1 compensated for reduction in liver IGF-1 in knockout mice [21]. The knockout mice had similar organ wet weight of liver, spleen, kidney, and heart with no difference in body and femoral lengths as the normal group [21]. This may suggest that spasticity or lowerlimb electrical stimulation may provoke muscular IGF-1 that compensates for reduced hepatic GH-IGF-1 axis after SCI. The observed protective mechanism of spasticity on muscle size, leg lean mass, and whole body composition is evoked by the autocrine/paracrine action of muscular IGF-1 [15]. Bickel et al. observed upregualtion of IGF-1 ribonucleic acid (RNA) following two bouts of electrical stimulation of the vastus lateralis muscle in individuals with SCI [22]. However, this is not a universal finding and others have noted that sex differences in load-induced myofiber hypertrophy among older adults cannot be explained by levels of circulating IGF-1 [23], a finding that may be explained by the effects of age on IGF-1 [24]. Future studies must investigate the expression of IGF-1 RNA and protein in individuals with SCI who do and do not have spasticity to delineate the source of plasma IGF-1.

In rat and human models, reduced IGF-1 has been associated with skeletal muscle atrophy and increased FM [14]. The mechanisms by which IGF-1 protect against skeletal muscle atrophy have been previously reviewed. Briefly, IGF-1 upregulates multiple intracellular signals that inhibit the secretion of several ubiquitinproteins, including atrogin-1, FOX-1 and MuRF1, that have been suggested as primary factors evoking skeletal muscle atrophy [1]. For example, administration of low doses of oral baclofen led to an increase in circulating plasma IGF-1 [25]. Surprisingly and against the proposed hypothesis, oral baclofen did not attenuate the protective effects of spasticity on body composition or glucose tolerance [10]. Moreover, oral baclofen was inversely related to the homeostatic model of insulin resistance [10]. These findings suggest that provoking IGF-1 secretion could help to attenuate the decline in muscle size after SCI. The maintenance of muscle size could facilitate glucose diffusion and improve glucose tolerance in individuals with SCI, as was previously documented [911]. Vincent et al. showed that IGF-1 serves as a neuroprotective mediator by preventing glutamate programmed cell death and promoting the survival of motor neurons in the spinal cord of embryonic rats [26].

The significance of the current work relies on the fact that understanding the pathway(s) by which spasticity affects the musculoskeletal system may lead to exploration of new interventions or tools to manage spasticity down the road. The pathway of the current findings is still debatable; it is possible that periodical hypertonicity evokes the release of IGF-1, which in turn can cause skeletal muscle hypertrophy via its action on the progenitor satellite cells [15]. IGF-1 has been shown to play a 
key role in muscle development and regeneration. Another interesting thought is that individuals with spastic SCI may experience repeated bouts of increased plasma IGF-1 after administration of oral baclofen [25]. IGF-1 was upregulated 3-7 d after botulinum toxin A injection to the gastrocnemius muscle of the rats. The upregulation of IGF-1 enhances the release of myogenic factors that, in turn, could activate the acetylcholine receptors and trigger spasticity [27].

The small sample size of the current study may have resulted in false positives (type 1 errors). However, this preliminary study should be viewed as a hypothesisgenerating work for future studies on the physiological effect of spasticity on body composition and metabolic profile. Further studies are warranted to investigate the metabolic role of spasticity in total IGF-1 and free IGF-1. Moreover, the current study primarily relied on plasma IGF-1 rather than muscle biopsy to measure protein or messenger RNA expressions [22]. Plasma IGF-1 was primarily used to establish the relationships with spasticity and muscle size; however, future studies should determine the effects of spastic muscles on IGF-1 expression. The study was limited to individuals with motor complete SCI (cervical 5 through thoracic 11) who experienced spasticity and were on antispastic medications for a specific time to ensure stability of the score. The inclusion of this level injury was based on the notion that a level of injury below thoracic 11 may be accompanied by denervation of the skeletal muscles, which leads to flaccid paralysis. Because bigger individuals may have larger muscle mass and, hence, more tonicity, we adjusted for whole body lean mass to control for the size effect; however, the relationship between spasticity and skeletal muscle size is still noted. The cross-sectional design of the current study does not establish causality, but rather offers a plausible explanation to an observation that was previously documented.

\section{CONCLUSIONS}

The current work lends credence to the hypothesis that maintaining muscle mass via spasticity or electrical stimulation resistance training may have beneficial effects on body composition and insulin sensitivity and thereby improve health after SCI [8-9,16]. In summary, spasticity appears to be associated with a protective effect on skeletal muscle size in individuals with motor complete SCI. The relationship between spasticity and muscle size can partially be explained by optimization of the IGF-1 profile, which is known to be associated with a protective effect against the deleterious adaptations in body composition after SCI. These findings warrant further studies that objectively evaluate spasticity and longitudinally determine the changes in skeletal muscle size and body composition after SCI. The findings do not advocate for removing antispasticity medications that manage the negative effects of spasticity, but rather support achieving a balance to maintain spasticity's positive effects.

\section{ACKNOWLEDGMENTS}

\section{Author Contributions}

Study concept and design: A. S. Gorgey, D. R. Gater.

Acquisition of data: A. S. Gorgey.

Analysis and interpretation of data: A. S. Gorgey, D. R. Gater.

Drafting of manuscript: A. S. Gorgey, D. R. Gater.

Critical revision of manuscript for important intellectual content: A. S. Gorgey, D. R. Gater.

Administrative, technical, or material support: D. R. Gater.

Study supervision: A. S. Gorgey.

Financial Disclosures: The authors have declared that no competing interests exist.

Funding/Support: This material was unfunded at the time of manuscript preparation.

Additional Contributions: The authors would like to thank all the participants who volunteered in the current study.

Institutional Review: All participants signed an informed consent statement approved by the local ethics committee.

Participant Follow-Up: The authors do not plan to inform participants of the publication of this study because contact information is unavailable.

\section{REFERENCES}

1. Qin W, Bauman WA, Cardozo C. Bone and muscle loss after spinal cord injury: Organ interactions. Ann N Y Acad Sci. 2010;1211:66-84. [PMID:21062296] http://dx.doi.org/10.1111/j.1749-6632.2010.05806.x

2. Adams MM, Hicks AL. Spasticity after spinal cord injury. Spinal Cord. 2005;43(10):577-86. [PMID:15838527] http://dx.doi.org/10.1038/sj.sc.3101757

3. Biering-Sørensen B, Kristensen IB, Kjaer M, BieringSørensen F. Muscle after spinal cord injury. Muscle Nerve. 2009;40(4):499-519. [PMID:19705475] http://dx.doi.org/10.1002/mus.21391 
4. Gorgey AS, Dudley GA. Skeletal muscle atrophy and increased intramuscular fat after incomplete spinal cord injury. Spinal Cord. 2007;45(4):304-9. [PMID:16940987]

5. Bauman WA, Spungen AM. Coronary heart disease in individuals with spinal cord injury: Assessment of risk factors. Spinal Cord. 2008;46(7):466-76. [PMID:18180789] http://dx.doi.org/10.1038/sj.sc.3102161

6. Bauman WA, Spungen AM. Metabolic changes in persons after spinal cord injury. Phys Med Rehabil Clin N Am. 2000;11(1):109-40. [PMID:10680161]

7. Bauman WA, Zhang RL, Spungen AM. Provocative stimulation of growth hormone: A monozygotic twin study discordant for spinal cord injury. J Spinal Cord Med. 2007; 30(5):467-72. [PMID:18092562]

8. Gorgey AS, Dudley GA. Spasticity may defend skeletal muscle size and composition after incomplete spinal cord injury [published correction appears in Spinal Cord. 2008; 46(12):825]. Spinal Cord. 2008;46(2):96-102.

[PMID:17637764]

http://dx.doi.org/10.1038/sj.sc.3102087

9. Gorgey AS, Chiodo AE, Zemper ED, Hornyak JE, Rodriguez GM, Gater DR. Relationship of spasticity to soft tissue body composition and the metabolic profile in persons with chronic motor complete spinal cord injury. J Spinal Cord Med. 2010;33(1):6-15. [PMID:20397439]

10. Gorgey AS, Chiodo AE, Gater DR. Oral baclofen administration in persons with chronic spinal cord injury does not prevent the protective effects of spasticity on body composition and glucose homeostasis. Spinal Cord. 2010;48(2): 160-65. [PMID:19687797] http://dx.doi.org/10.1038/sc.2009.105

11. Bennegard GM, Karlsson AK. Higher glucose uptake in paralysed spastic leg. Spinal Cord. 2008;46(2):103-6. [PMID:17579617] http://dx.doi.org/10.1038/sj.sc.3102083

12. Löfvenmark I, Werhagen L, Norrbrink C. Spasticity and bone density after a spinal cord injury. J Rehabil Med. 2009;41(13):1080-84. [PMID:19894005] http://dx.doi.org/10.2340/16501977-0469

13. Franzoi AC, Castro C, Cardone C. Isokinetic assessment of spasticity in subjects with traumatic spinal cord injury (ASIA A). Spinal Cord. 1999;37(6):416-20.

[PMID:10432261]

http://dx.doi.org/10.1038/sj.sc.3100849

14. Adams GR. Role of insulin-like growth factor-I in the regulation of skeletal muscle adaptation to increased loading. Exerc Sport Sci Rev. 1998;26:31-60. [PMID:9696984] http://dx.doi.org/10.1249/00003677-199800260-00004

15. Adams GR. Invited Review: Autocrine/paracrine IGF-I and skeletal muscle adaptation. J Appl Physiol. 2002;93(3): 1159-67. [PMID:12183514]
16. Gorgey AS, Mather KJ, Cupp HR, Gater DR. Effects of resistance training on adiposity and metabolism after spinal cord injury. Med Sci Sports Exerc. 2012;44(1):165-74.

[PMID:21659900]

http://dx.doi.org/10.1249/MSS.0b013e31822672aa

17. Sacheck JM, Hyatt JP, Raffaello A, Jagoe RT, Roy RR, Edgerton VR, Lecker SH, Goldberg AL. Rapid disuse and denervation atrophy involve transcriptional changes similar to those of muscle wasting during systemic diseases. FASEB J. 2007;21(1):140-55. [PMID:17116744] http://dx.doi.org/10.1096/fj.06-6604com

18. Gorgey AS, Mather KJ, Gater DR. Central adiposity associations to carbohydrate and lipid metabolism in individuals with complete motor spinal cord injury. Metabolism. 2011;60(6):843-51. [PMID:20870252] http://dx.doi.org/10.1016/j.metabol.2010.08.002

19. Harris RL, Putman CT, Rank M, Sanelli L, Bennett DJ. Spastic tail muscles recover from myofiber atrophy and myosin heavy chain transformations in chronic spinal rats. J Neurophysiol. 2007;97(2):1040-51. [PMID:17122320] http://dx.doi.org/10.1152/jn.00622.2006

20. Rao U, Shetty KR, Mattson DE, Rudman IW, Rudman D. Prevalence of low plasma IGF-I in poliomyelitis survivors. J Am Geriatr Soc. 1993;41(7):697-702. [PMID:8315177]

21. Yakar S, Liu JL, Stannard B, Butler A, Accili D, Sauer B, LeRoith D. Normal growth and development in the absence of hepatic insulin-like growth factor I. Proc Natl Acad Sci USA. 1999;96(13):7324-29. [PMID:10377413] http://dx.doi.org/10.1073/pnas.96.13.7324

22. Bickel CS, Slade JM, Haddad F, Adams GR, Dudley GA. Acute molecular responses of skeletal muscle to resistance exercise in able-bodied and spinal cord-injured subjects. J Appl Physiol. 2003;94(6):2255-62. [PMID:12611774]

23. Bamman MM, Hill VJ, Adams GR, Haddad F, Wetzstein CJ, Gower BA, Ahmed A, Hunter GR. Gender differences in resistance-training-induced myofiber hypertrophy among older adults. J Gerontol A Biol Sci Med Sci. 2003;58(2): B108-16. [PMID:12586847] http://dx.doi.org/10.1093/gerona/58.2.B108

24. Juul A, Kastrup KW, Pedersen SA, Skakkebaek NE. Growth hormone $(\mathrm{GH})$ provocative retesting of 108 young adults with childhood-onset GH deficiency and the diagnostic value of insulin-like growth factor I (IGF-I) and IGF-binding protein-3. J Clin Endocrinol Metab. 1997;82(4): 1195-201. [PMID:9100596]

http://dx.doi.org/10.1210/jc.82.4.1195

25. Bauman WA, Kirshblum SC, Morrison NG, Cirnigliaro CM, Zhang RL, Spungen AM. Effect of low-dose baclofen administration on plasma insulin-like growth factor-I in persons with spinal cord injury. J Clin Pharmacol. 2006; 46(4):476-82. [PMID:16554457] http://dx.doi.org/10.1177/0091270006286641 
26. Vincent AM, Mobley BC, Hiller A, Feldman EL. IGF-I prevents glutamate-induced motor neuron programmed cell death. Neurobiol Dis. 2004;16(2):407-16.

[PMID:15193297]

http://dx.doi.org/10.1016/j.nbd.2004.03.001

27. Shen J, Ma J, Lee C, Smith BP, Smith TL, Tan KH, Koman LA. How muscles recover from paresis and atrophy after intramuscular injection of botulinum toxin A: Study in juvenile rats. J Orthop Res. 2006;24(5):1128-35. [PMID:16602109]

http://dx.doi.org/10.1002/jor.20131

Submitted for publication April 25, 2011. Accepted in revised form September 19, 2011.
This article and any supplementary material should be cited as follows:

Gorgey AS, Gater DR. Insulin growth factors may explain relationship between spasticity and skeletal muscle size in men with spinal cord injury. J Rehabil Res Dev. 2012;49(3):373-80.

http://dx.doi.org/10.1682/JRRD.2011.04.0076

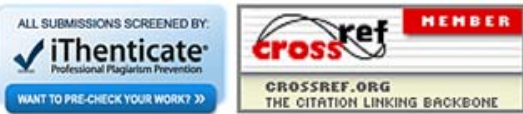

